DESY M-83-17

May 1983

\title{
WAKE FORCE COMPUTATION IN TIME AND FREQUENCY DOMAIN FOR CYLINDRICALLY SYMMETRIC STRUCTURES EXCITED BY BUNCHES OF ELECTRONS AT THE SPEED OF LIGHT
}

by

T. Weiland

Paper presented at the IV-th COMPUMAG Conference Genoa, 1983 
DESY behält sich alle Rechte für den Fall der Schutzrechtserteilung und für die wirtschaftliche Verwertung der in diesem Bericht enthaltenen Informationen vor.

DESY reserves all rights for commercial use of information included in this report, especially in case of filing application for or grant of patents.

"Die Verantwortung für den Inhalt dieses Internen Berichtes liegt ausschließlich beim Verfasser" 
ABSTRACT

In high energy electron/positron accelerators and storage rings intense bunches of charged particles pass: cylindricially symmetric structures at almost the sprest of light. Ihese drarges excile electromagnetic self fields having in general all six components. The induced fields produce highly nonlinear self forces in all three spatial directions which do limit the performance of most of existing and planned high eneryy machines. Two complementary approaches are presented for the computation of the electromagnetic ficids in either time or frequency domain by means of a mesh method allowing for arbitrarily shaped geometries. Results of these computer codes represent a key clement in analysis and prediction of performance for future high energy facilities.

\section{SUMMARY}

Maxwell's Equations in arbitrarily inhomogeneous media can be transformed by means of a general mesh method into an equivalent set of matrix equations [1]. The solution of the matrix problem yields directly the electromagnetic field components as a function of time and spatial coordinates. The matrix formalism enables the solution of time dependent and steady state problems and can be applied to arbitrarily shaped structures in two and three dimensions. Many complicated problems have been solved by this method in the past: Three dimensional eddy currents in laminated $[2,3]$ and/or solid $[4]$ inetallic bodies and wave propagation on lossy vave guides [5].

Here we want to deal with free moving charges in accelerating structures and their self excited electromagnetic fields. Such problems occur in any linear or circular accelerator. Self excited fields were found to establish a serious performance limitation to many acccelerators and they are likely to be even more dangerous for future large facilities presently under study.

The electromagnetic interaction between the particles and the environment can be looked at in two equivalent regimes: time and frequency domain [6]. However for practical computations the two regimes are used for different purposes: The time domain is adequate for all transient effects that do not last very long - specifically not long enough to show resonant steady state behaviour - Frequency domairi calculations are superior for considering effects with a long memory.

The general Maxwell matrix set of equations has been applied to both regimes [7-10] and allows a complete analysis of accelerator components by means of two computer codes URMEL [11] and TBCI [12]. These codes have now been extensively applied to almost all existing and future high energy accelerator structures. The time domain version allows for graphical output on $16 \mathrm{~mm}$ movie.
THE PHYSICAL PROBLEM

For accelerating particles to high energies one uses high frequency fields in resonators with small side tubes for letting the particles traverse the resonator such that they pick up energy from the externally supplied field energy. Figure la shows the most simple form of a cylindrically symmetric "pill-box" cavity with a sketch of the electric field of the lowest resonant TMO10 mode. In order to bring the external r.f. energy to the beam efficiently different geometries have been developed and fig. ib shows the most common "nose cone" type of accelerating cavity - due to the symmetry only one a quarter has to be considered - . These fields are externally excited but additional fields are generated by the charged particles when they pass this cavity. In order not to confuse the situation we consider these two types of fields separate.

Figure 2 shows the electric fields ( $r \cdot H_{\varphi}=$ const.) as a function of time excited by a Gaussian line charged bunch traversing on the axis three accelerating cavities. At the beginning all cavities are empty. Energy is radiated by particles and after they have left a cell the remaining energy partly stays inside - in form of resonating modes - and partly propagates into the beam pipes on both ends.

The interesting quantity affecting each single particle in it's motion is the electromagnetic force excited by the ensemble. It is convinient to introduce a comoving system and to describe all quantities affecting the beam in these comoving coordinates. Figure 3 shows the longitudinal force acting on three particles in the middle of the bunch and at $2 \sigma$ apart during the passage of a single cell of a PETRA cavity.

Integrating these forces over the passage time for each position inside or behind the ensemble yields the so called wake potential[13]. It is sufficient for many cases to consider only these time averaged forces since particle motion is slow compared to the time scale in which the forces vary. The time argument now has disappeared and the variable is the relative particle positions. Each value in fig. 4 represents a result of an integration of the time dependent transient forces as shows in fig. 3. As one can see from figure 4 the wake potential. shows a resonant character behind the exciting bunch. This reflects the

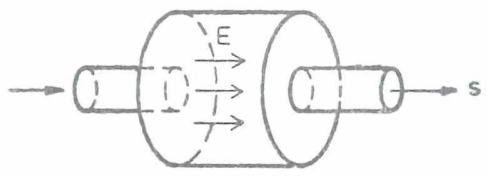

Fig. 1.

a) "Pill-box" cavity with side tubes for particle acceleration with the TMO10 mode

b) optimized shape of the PETRA accelerating cavity and $r \cdot|\vec{E}|$ lines $(r \cdot H \varphi=$ const. $)$

of the TMO10 mode at $500 \mathrm{MHz}$

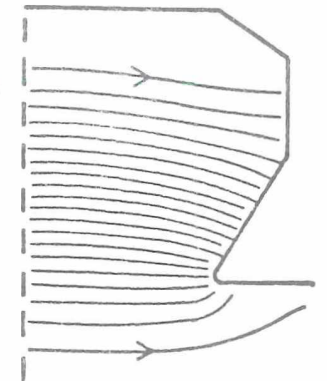



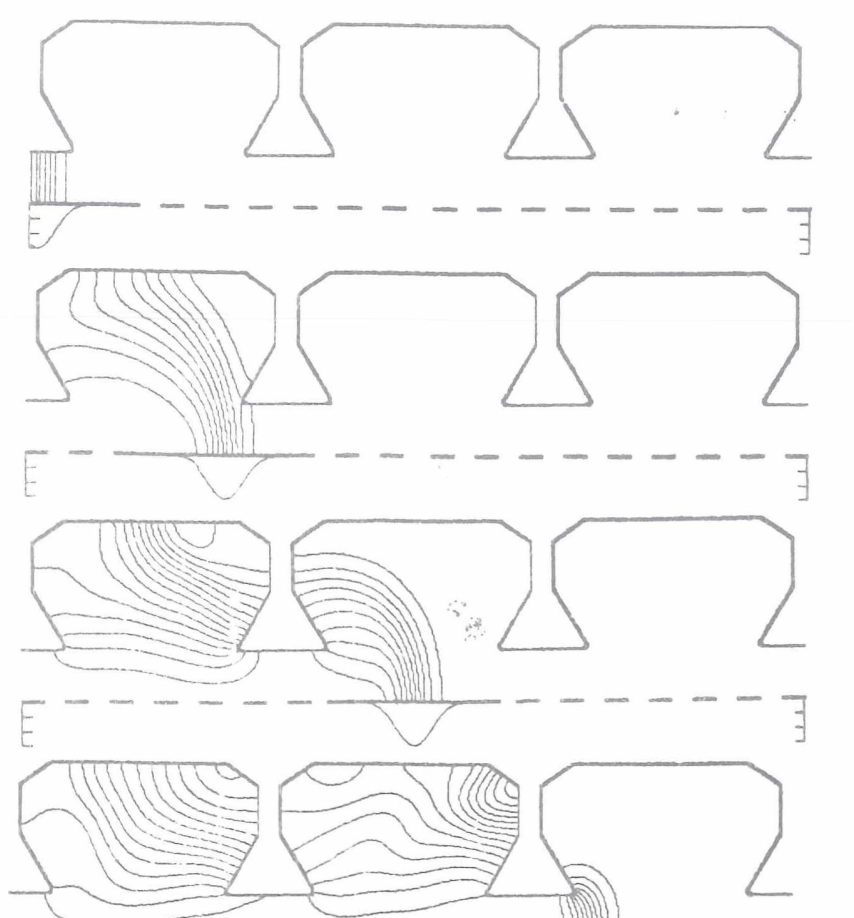

E - - - - - - -

Fig. 2. Gaussian bunch $(\sigma=2 \mathrm{~cm})$ traversing at the speed of light an accelerating PETRA cavity

( 3 cells) on axis (shown are lines of $I \bullet H \varphi=$ const.)

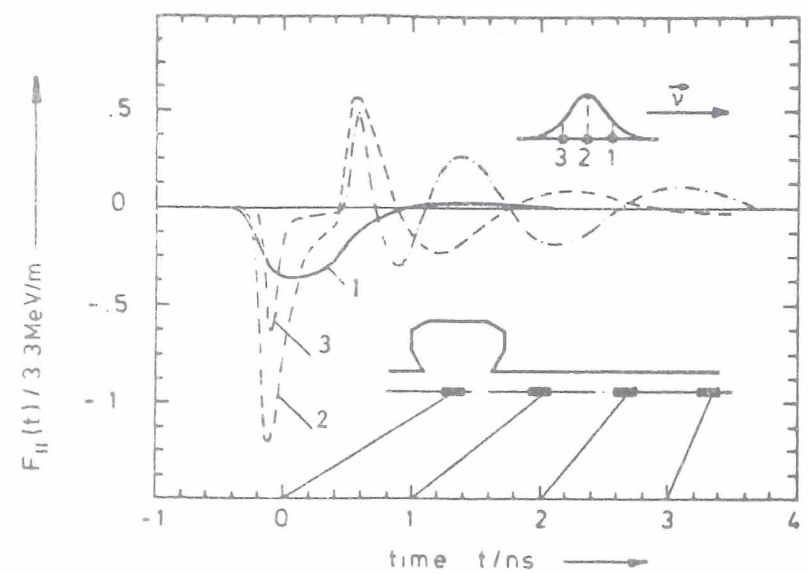

Fig. 3. Decelcrating longitudinal self force $F_{H}(t)$ experienced by the three particles $(1,2,3)$, two $\sigma$ in front of the bunch center, at the center and two $u$ behind the center during the passage of a single PI.TRA cavily cell with an adjacent long side tube. At time zero the bunch center is the middle of the cell. The bunch length $\sigma$ is $3 \mathrm{~cm}$ the total charge is $1 \mu \mathrm{C}$. The cell length is $30 \mathrm{~cm}$ ( $\triangleq 1 \mathrm{~ns}$ ). Note how long it takes until all fields have reached the particles.

visible field character in fig, 2 where the fields near the beam look very much different from resonant modes whereas after the passage the remaining part looks very much like a combination of a few resonant modes. Although the entire wake potential (not the transient wake force $F(t) 1$ ) can be computed by either frequency or time domain methods it happens that thousands of modes would have to be computed for the wake potential inside the bunch whereas just a few modes suffice for positions well behind the bunch center $[6]$.
Electromagnetic interactions between particles inside an ensemble thus are adequately described by the time domain. Interactions between one bunch and a second one following much later are better described by the resonant modes with a long memory. Accordingly these different regimes are called short range (high frequency) and long range (low frequency).

\section{TIME DOMAIN COMPUTATIONS}

Applying the generalized [1] Yee algorithm [14] in a rectangular mesh and. replacing the unknown components of $\vec{E}$ and $\vec{B}$ by the column vectors $\vec{b}$ and $\vec{b}$ holding the values of $\bar{E}$ and $B$ at the grid nodes yields the matrix replacement for Maxwell's equations [7]:

$$
\begin{aligned}
& R \cdot \vec{e}=-\mu_{0} \vec{h}, \\
& 2 \\
& R \cdot \vec{h}=\varepsilon \vec{e}+\vec{j} .
\end{aligned}
$$

In order to allow large node numbers $\left(>10^{5}\right)$ we choose the explicit "leap frog" scheme for discretization of the time dependance:

$f^{n}=f(n \cdot \Delta t) ; \quad \dot{f}^{n}=\frac{1}{\Delta t}\left(f^{n+1 / 2}-f^{n-1 / 2}\right)$.

Maxwell's equation are then rearranged into the "update" form:

$e^{\vec{n}+1 / 2}=e^{\vec{n}-1 / 2}+\frac{\Delta t}{\varepsilon_{0}}\left(\stackrel{\imath}{R} \vec{h}^{n}-\vec{j}^{n}\right)$,
$\vec{h}^{n+1}=h^{n} \quad-\frac{\Delta t}{\mu_{0}} R \quad \vec{e}^{n+1 / 2}$.

(For simplicity we assumed here a homogenous vacuum but the algorithm and most of the programs allow for arbitrarily shaped distributions of permeability, permittivity and conductivity). The driving term $\vec{j}$ is given by the moving particles and in a very simple time stepping procedure we may obtain from equations 4 and 5 the electromagnetic field at any time and any location inside the grid.

For the most important case of cyclindrically symmetric structures a computer code TBCI has been developed that calculates fields of any azimuthal dependance ( $\propto \cos m \varphi, m=0$ for decelerating longitudinal fields, $m=1,2 \ldots$ for deflecting fields) as a function of time, see e.9. figures 2,5 . Transverse fields are excited when a particle beam traverses a cavity off axis.

Since wake potentials can be computed from resonant modes only for a very specific type of cavity geometry $[15,16]$ (which happens to fit the Bessel function approach) and only very few modes can be obtained from mesh codes for realisticly shaped structures [10,17 ], TBCI seems to be the only tool to attack the general task of computing electromagnetic interactions in resonant and nonresonant geometries.

One more typical example is shown in fig. 5. Bellow sections as shown there are used to compensate for thermal elongation of adjacing components. (This is obviously a hard problem to solve with a resonant mode approach).

TBCI results for all important accelerator components may be summed and used to solve separately for the beam dynamics problems by means of particle simulation codes [18] For these purposes grids with over 200.000 nodes have been used on an IBM 3081. Typical. cpu time ranges from few seconds to 4 hours and the average cpu time scales like $\mathrm{N} 3 / 2$ with $\mathrm{N}$ as the number 


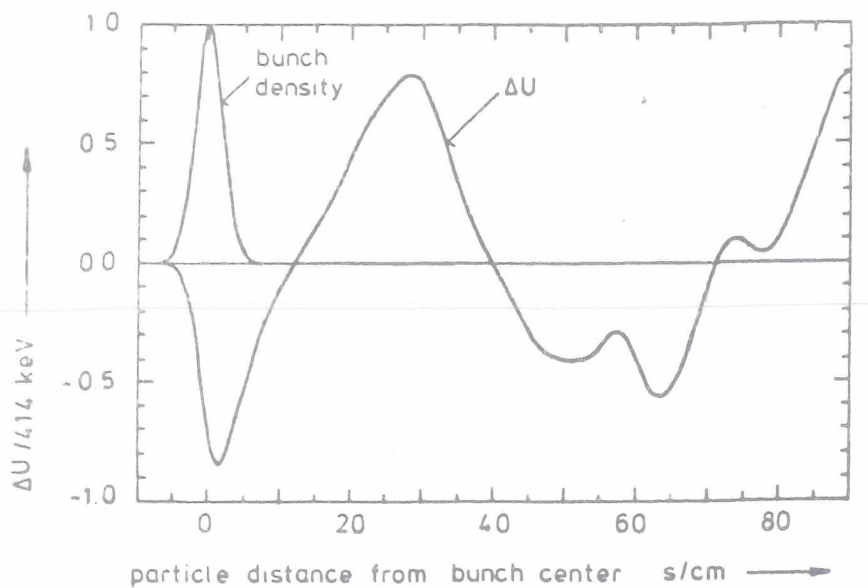

Fig. 4. Energy loss $U$ of particles inside and behind a short Gaussian bunch $(\sigma=2 \mathrm{~cm}, Q=1 \mu \mathrm{C})$ after the passage of a single PETRA cavity cell. Leading particles are on the left. For $-10 \mathrm{~cm}<s<10 \mathrm{~cm}$ the wake potential is of non resonant character and is resonant for $s>10 \mathrm{~cm}$

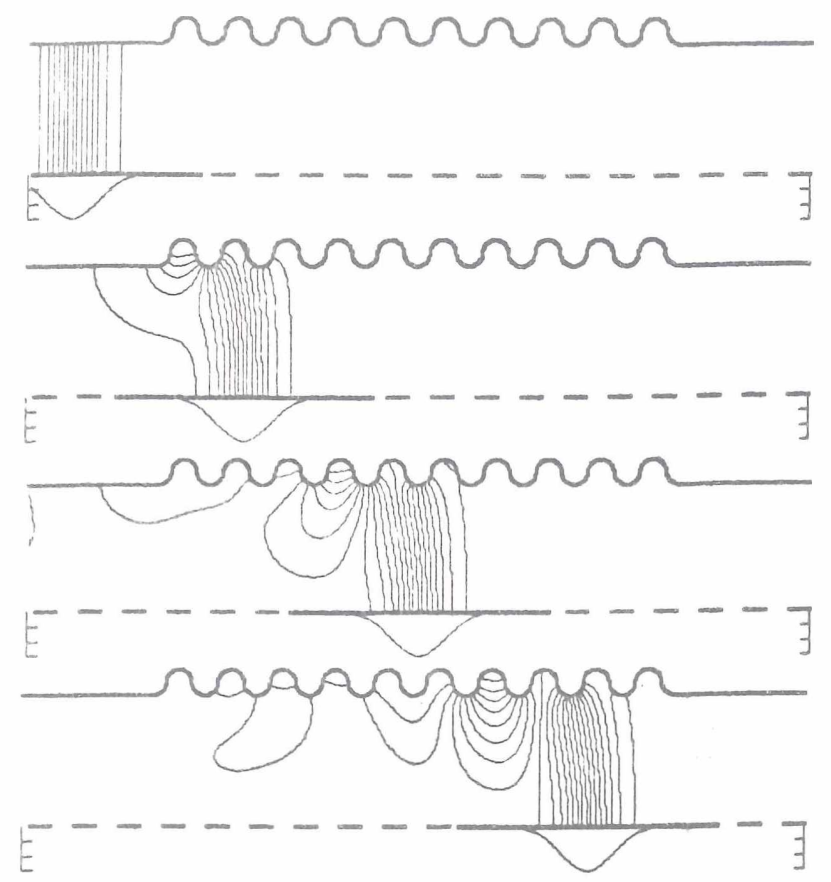

Fig. 5. Electric field of a Gaussian bunch $(\sigma=1 \mathrm{~cm})$ passing a bellow section at the speed of light

of grid nodes. The rectangular grids are very flexible by allowing triangular sub cells but a realistic mesh cannot be shown in little space here. The flexibility in the grid will be shown later in frequency domain codes which need only a few thousand nodes. The fully 3 D version presently being coded will be able to handle one order of magnitude larger grids with over 106 nodes.
FREQUENCY DOMAIN

The first task one meets in frequency domain is the optimization of an accelerating cavity in order to transfer of energy in the most efficient way to the particle beam. Computer codes for this task have to solve an eigenfrequency problem (e.g. SUPERFISH [17] and many others listed in ref. 10). However most of these codes compute only cylindrically symmetric fields but the beam also excites higher transverse resonant modes in these cavities. In fact most of the beam dynamics problems are caused by deflecting modes. The problem is solved by using the general form of Maxwell's matrix equations (1) and (2) and by replacing the time derivative by assuming harmonic time dependance:

$$
\begin{aligned}
& R \cdot \vec{e}=-i \omega \mu_{0} \vec{h}, \\
& \vec{R} \quad \vec{h}=i \omega \varepsilon_{0} \vec{e} .
\end{aligned}
$$

One may eleminate either e or $h$ to obtain:

$$
\text { or } \begin{aligned}
R \hat{R} \vec{h} & =\left(\omega_{c} / c\right)^{2} \vec{h}, \\
& \tilde{R} R \vec{e}=\left(\omega_{0} / c\right)^{2} \vec{e} .
\end{aligned}
$$

It is important to notice that the obtained eigenvalue matrix problems are linear and that the algebraic eigenvalue is the frequency squared (this is in general not the case especially not if one uses a mixed set of electric and magnetic unknowns yielding nonlinear eigenvalue probelms - see ref. 19 and references in ref. 10 - and not the set of purely electric or magnetic field components used in URMEL [10-11] and in the general formulation of Maxwell's eigenvalue problems $[1,5])$. For $2.5 \mathrm{D}$ geometries the computer code URMEL [10-11] computers up to 50 eigensolutions in automatically given correct order (ascending in frequency). The mesh definitions and input data are almost compatible with TBCI and thus one and the same set of input data is used to analyse time and frequency domain fields. The eigenvalue solver is a simple matrix multiplication algorithm plus a Rayleigh expression which yields reasonable convergence.

Figure 6 shows a typical grid with triangular subcells resulting in a good shape approcimation. A few results found for other typical accelerating cavities are shown in fig. 7 (new PETRA copper cavity) and fig. 8 (new superconducting cavity of single mode type SMC).

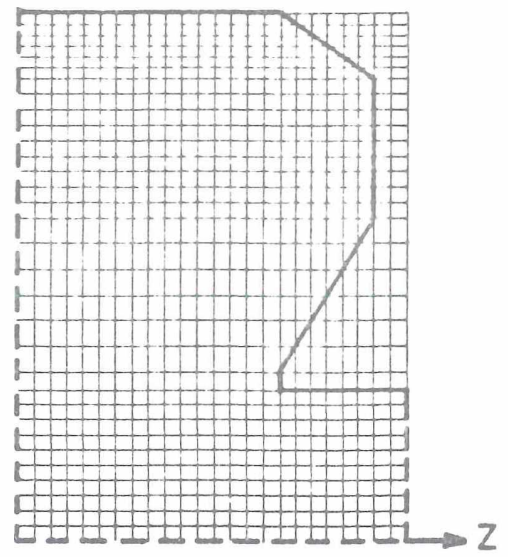

Fig. 6. Typical grid with irregular step sizes as used for approximation of PETRA cavity. Note the good approximation by triangular sub cells 


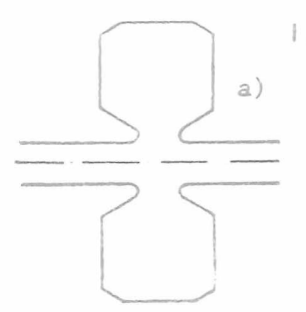

ig. 7. I ighl'Lh transverse deflecting mode in the new PETRA accelerating cavity at $f=2278 \mathrm{MHz}$ with fields $\propto \cos \varphi$

a) full cell geometry

b) electric field at $\varphi=0$

c) magnetic field at $\varphi=90^{\circ}$
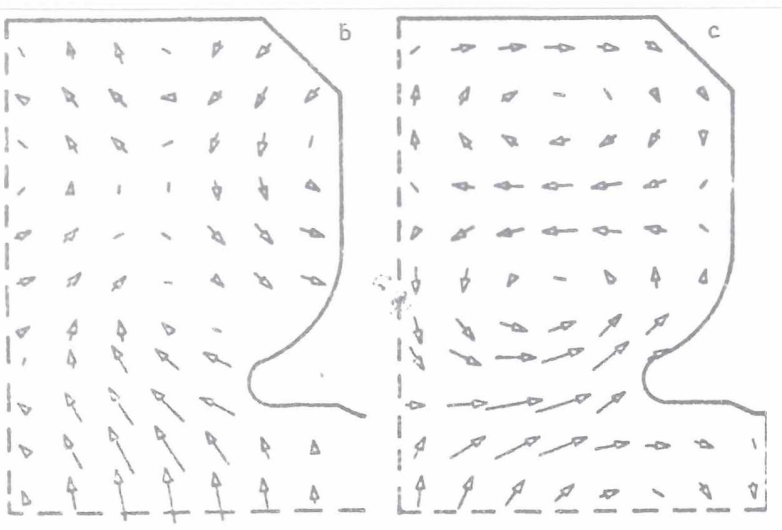

CONCLUSIONS

The complementary approaches of time and frequency domain are both realized by computer codes based on one and the same matrix set of equations which replace Maxwell's equations in a general orthogonal grid. Transient electromagnetic forces may be obtained as well as resonant modes and thus a complete analysis of accelerator components is possible. Especially for future accelerators one now may optimize accelerating geometries in a much wider sight with one single set of codes. Although these programs have been used for many specific problems occurring in accelerator study many other problems could be solved as well - e.g. antenna radiationPresent research is focussed on improving the 3 D eigenvalue solver procedure and on the use of very large grids ( $>10^{6}$ modes) in tim domain.

[1] T. Weiland:" A Discretization Method for the solutionof Maxwell's Equations for Six Component Fields" Electronics and Communication AEÜ 31 (1977), pp. 116-120.

[2] T.Weiland: "On the Calculation of Eddy Currents in Arbitrarily shaped Three Dimensional Laminated Iron Cores, Part I: The Method" Archiv für Elektrotechnik AfE 60 (1978), pp. 345-351.

[3] H. Euler and T. We iland: "On the Calculation of Eddy Currents in Arbitrarily Shaped Three Dimensional Laminated Iron Cores, Part II Applications" AfE 61 (1979), pp.103-109

[4] T. Weiland:"On the Calculation of Eddy Currents in Solid, Three Dimensional Arbitrarily Shaped Iron Cores" etz Archiv 1 (1979), pp. 263-267.

[5] T. Weiland:"Lossy Waveguides with an Arbitrary Boundary Contour and Distribution of Material" Electronics and Communications AEÜ 33 (1979), pp. 170-174

[6] T. Weiland and B. Zotter: "Wake Potentials of a Realistic Current in a Cavity" Particle Accelerators 11 (1981), pp, 143-151

[7] T.Weiland:"Transient Electromagnetic Fields Excited by Bunches of charged Particles in Cavities of Arbitrary Shape". Proceedings of the XI-th International Conference of High Energy Accelerators, Geneva 1980, Birkhäuser Verlag, edited by W.S. Newman, pp. 570-575.

[8] T. Weiland: "Transverse Beam Cavity Interaction, Part I: Short Range Forces" DESY 82(015) 1982
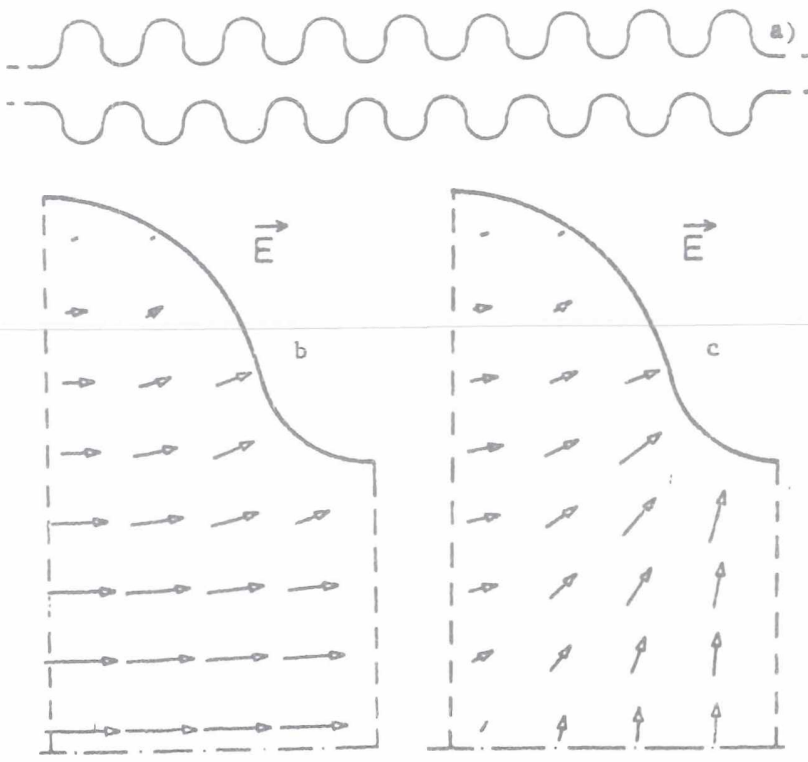

Fig. 8. Electric field of the accelerating mode and the only deflecting mode in the Single Mode Cavity (SMC).

This cavity is specially designed to have only these two resonant modes. Higher frequencies propagate into the side tubes and cannot resonate.

a) multi cell structure

b) computed lowest mode without azimuthal field dependence $f=1001 \mathrm{MHz},(0$-mode, TMO1O)

c) deflecting mode with field $\propto \cos \varphi ; f=1281 \mathrm{MHz}$

[9] T. Weiland:" Comment on Wake Field Computation in Time Domain" accepted for publication in: Nuclear Instruments and Methods

[10] I. Weiland: "On the Computation of Resonant Modes in Cylindrically Symmetric Cavities" accepted for publication in: Nuclear Instruments and Methods

[11] T. Weiland:"URMEL user Guide" DESY M82-24 (1982)

[12] T, Weiland:"BCI - A Computer Program for Transient Electromagnetic Fields of Bunched Beams in Accelerators" CERN/ISR-TH/80-45, 1980

[13] K.L.F. Bane, J.B. Styles and P.B. Wilson:" "Comparison of Measured and Computed ioss to Parasitic Modes in Cylindrical Cavities with Beam Ports" Proceedings of the 1977 Particle Accelerator Conference, Chicago and SLAC-Pub-1908

[14] K.S. Yee:"Numerical Solution of Initial Boundary Value Problems Involving Maxwell's Equations in Isotropic Media" IEEE, AP-14 (1966), Pp. 302-307

[15] E. Keil: "Diffraction Radiation of Charged Particles Moving in a Corrugated Cylindrical Pipe" Nuclear Instruments and Methods 100 (1972), pp. 419-427

[16] K.L.F. Bane and B. Zotter:"Transverse Modes in Periodic Cylindrical Cavities", see ref. 7, pp. 581-585

[17] K. Halbach and R. F. Holsinger:" SUPERFISH - a Computer Program for Evaluation of RF Cavities with Cylindrical Symmetry" Particle Accelerators 7 (1976), pp. 213-222

[18] T. Weiland: "On the Quantitative Prediction of Bunch Lengthening in High Energy Electron Storage Rings", DESY 81 (088), 1981

[19] R.L. Gluckstern, R.F. Holsinger and G.N. Minerbo: "ULTRAFISH- Generalization of SUPERFISH to $m>l$ " Proceedings of the 1981 Linear Accelerator Conference in Los Alamos, LA-9234C. edited by R.A. Jameson and L.S. Taylor, Los Alamos National Laboratory. 